\title{
The Effect of Spinopelvic Motion on Implant Positioning and Hip Stability Using the Functional Safe Zone of THR
}

\author{
Nathanael Heckmann, Nicholas A. Trasolini, \\ Michael Stefl, and Lawrence Dorr
}

\section{Key Points}

- The Lewinnek safe zone has failed in its predictive limits of cup position for stability.

- The functional hip motion safe zone is measured by the combined sagittal index (CSI) and is the best measure of risk of impingement including dislocation.

- Excessive femoral motion measured by pelvic femoral angle (PFA) is the greatest reason for dislocation, not the acetabular position.

- Preoperative sagittal X-rays should be taken to determine if spinopelvic imbalance is present and if so the cup position is adapted to the imbalance. Postoperative sagittal X-rays will confirm the hip is safe from spinopelvic to hip impingement.

- Intraoperatively ideally the technique uses combined anteversion rather than just anteversion and targets the cup position with a smart tool for precision so the chance for the hip being in the functional safe zone is optimized.

N. Heckmann · N. A. Trasolini

Keck Medical Center of USC, Los Angeles, CA, USA e-mail: Nicholas.Trasolini@med.usc.edu

M. Stefl

McFarland Clinic, Ames, IA, USA

L. Dorr $(\bowtie)$

Pasadena, CA, USA

\subsection{Introduction}

Accurate and precise component positioning in total hip arthroplasty is a ubiquitous goal amongst hip surgeons and an important topic of research. Early studies defined a "safe zone" for placement of the acetabular cup, and divergence from the defined safe zone was shown to predispose patients to dislocation [1]. However, ideas regarding the position of the acetabular cup have continued to evolve beyond the initial description by Lewinnek in 1978 [1]. Murray et al. [2], in 1993, defined anatomic, operative, and radiographic parameters for inclination and anteversion. DiGioa et al. expanded upon this work by describing functional cup position, rather than just anatomical inclination and anteversion, as being the angles of the acetabulum that correlated to the axis of the body, using lateral radiographic measurements of the spine, pelvis, and hip [3]. This expanded definition was the first to consider spinal parameters as part of a functional spine-pelvis-hip relationship. Lazennec et al. [4], in France, used a new imaging modality (EOS, Biospace Med, Paris, France) to clearly demonstrate the interrelationship of spinal mobility to acetabular position during postural change of sitting to standing (Fig. 12.1). This research increased our understanding that spine-pelviship motion is synchronized for the purpose of allowing the normal hip to move freely through its arc of motion without the greater trochanter 
Fig. 12.1 Illustration of normal motion of spine-pelvis-hip during postural change from stand to sit. On left is standing and pelvis tilts anteriorly with sacral slope of $35^{\circ}$. The pelvic incidence is low at $40^{\circ}$. The femur is in extension, but pelvic femoral angle not measured here (see Fig. 12.2). On the right is sitting position with pelvis tilted posteriorly and sacral slope is $20^{\circ}$. $\mathrm{PI}$ is static and remains at $40^{\circ}$. The femur flexes but not to $90^{\circ}$. Normal sitting is a combination of posterior tilt of the pelvis and flexion of the femur of $55^{\circ}-70^{\circ}$

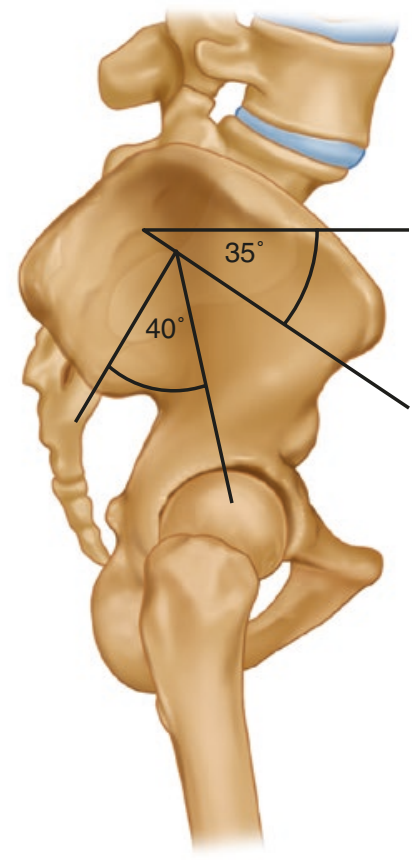

impinging on the pelvis, or the lesser trochanter on the ischium. As hip surgeons learned more about the anatomy of this spine-pelvis-hip relationship, research shifted to studying the effect of this relationship on outcomes following total hip arthroplasty. The cumulative effect of that work has been to redefine the safe zone for acetabular component positioning (functional safe zone) and the influence of sagittal hip motion by taking into account spinopelvic motion. This chapter will focus on the evolving definition of an acetabular safe zone in the context of the spine-pelvis-hip construct, as well as how to personalize and optimize component positioning based on patient-specific spinopelvic parameters.

\subsection{Normal Spine-Pelvis-Hip Motion}

Understanding the relationship between spinopelvic motion and total hip arthroplasty requires a familiarity with normal pelvic movement in the sagittal plane. When one stands, the pelvis is tilted anteriorly and the lumbar spine assumes a normal lordotic curvature (Fig. 12.1). This positions the acetabulum over the femoral head, while the extended hip allows the spine to support the load of the anterior trunk mass over the pelvis [5]. The amount of anterior pelvic tilt and lumbar spinal lordosis is dependent on a measurement named pelvic incidence (PI) defined by Legaye [6]. High pelvic incidence means that the lordosis and sacral tilt are increased. With postural change, these patients have increased pelvic motion and less hip motion. Low pelvic incidence leads to decreased sacral tilt and a more kyphotic lumbar spine. This means the hip must flex more when a patient moves from a standing to seated position resulting in an increased risk of impingement [5]. It is unknown why people have more or less standing pelvic tilt, but it has been suggested that patients with low pelvic incidence are at greater risk of arthritis of the hip. They definitely have more hip flexion and higher risk of impingement.

With sitting, the pelvis tilts posteriorly as the lordosis of the lumbar spine straightens (Fig. 12.1). This change accommodates the necessary hip flexion and internal rotation of the femur by opening the acetabulum by increasing the functional anteversion of the cup $[4,7,8]$. The spinopelvic motion from standing to sitting is normally $20^{\circ}$, while the 
femur flexes only $55^{\circ}-70^{\circ}$ to accomplish sitting [4, 9]. To bend forward from the waist to pick up an object on the floor requires increased flexion of the hip to $85^{\circ}$ combined with internal rotation of $12^{\circ}$ [10]. The magnitude of spinopelvic mobility affects the amount of hip motion needed to perform these activities. With increased mobility of the pelvis, the hip does not need to flex as much to sit or extend as much to stand. To the contrary, when the spinopelvic construct is stiff, the hip must flex more to sit or bend and extend more to stand. Increased hip motion increases the risk for impingement (articular and extra-articular) $[4,11]$.

Measurements can be made to quantify the mobility of the spinopelvic construct and hip before and after THA (Table 12.1). The radio- graphic measurements derive from the lateral standing and sitting spinopelvic X-rays (Figs. 12.1 and 12.2). The spinal segments from

Table 12.1 Normal radiographic spinopelvic values

\begin{tabular}{l|l|l|l}
\hline & Standing & Sitting & $\Delta$ \\
\hline Pelvic incidence & $53^{\circ} \pm 11^{\circ}$ & $53^{\circ} \pm 11^{\circ}$ & - \\
\hline Sacral tilt & $40^{\circ} \pm 10^{\circ}$ & $20^{\circ} \pm 9^{\circ}$ & $11^{\circ}-29^{\circ}$ \\
\hline $\begin{array}{l}\text { Pelvic femoral } \\
\text { angle }\end{array}$ & $180^{\circ} \pm 15^{\circ}$ & $125^{\circ} \pm 12^{\circ}$ & $50^{\circ}-75^{\circ}$ \\
\hline Anteinclination & $35^{\circ} \pm 10^{\circ}$ & $52^{\circ} \pm 10^{\circ}$ & - \\
\hline
\end{tabular}

$\Delta=$ difference between standing and sitting

Pelvic incidence is a static anatomic measurement and does change between standing and sitting. The other three measurements are dynamic positional parameters so they change between postural positions
Fig. 12.2 (a) Illustration of a pelvis that is fixed in anterior tilt or "stuck standing." The lumbar spine is in lordosis. The pelvic incidence is $45^{\circ}$ and the sacral slope is $40^{\circ}$ standing and only $35^{\circ}$ sitting which means it has only $5^{\circ}$ of motion which means the spine is effectively fused. The pelvic femoral angle is $170^{\circ}$ standing because of the anterior tilt, but with flexion goes to $115^{\circ}$ which is excessive flexion and with the pelvis fixed with anterior tilt the acetabulum remains relatively closed so this flexion presents risk for anterior impingement and posterior dislocation. (b) Illustration of pelvis that is fixed in posterior tilt or "stuck sitting." The spine is straight standing and actually slightly kyphotic sitting. The pelvic incidence is $45^{\circ}$ and fixed posterior tilt is more commonly associated with low pelvic incidence. The sacral slope goes from $20^{\circ}$ standing (with normal motion, the standing sacral slope is above $30^{\circ}$ ); the sitting sacral slope is $15^{\circ}$, so again this is only $5^{\circ}$ of motion which means the lower lumbar spine is effectively fused. With a stuck-sitting deformity, there is hyperextension of the femur and the PFA at standing is $210^{\circ}$. This creates risk of posterior impingement of the greater trochanter on the pelvis which presents some risk for anterior dislocation. With sitting there is normal flexion of the femur of $124^{\circ}$
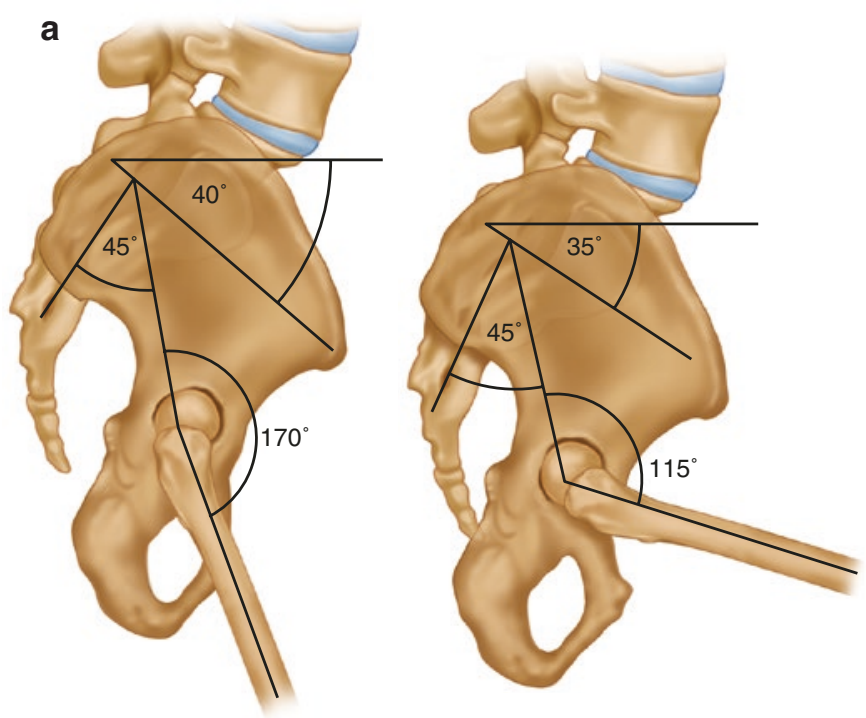

b
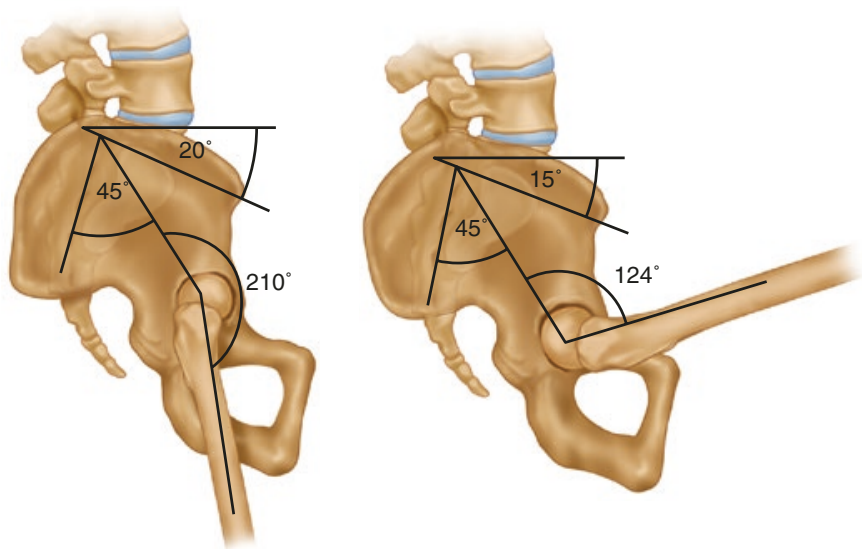
L3 to S1 need to be visualized because this segment of the lumbar spine moves with the pelvis. The sacrum is fixed to the lower lumbar spine, so degenerative disease of this portion of the spine impacts the mobility of the pelvis $(\Delta \mathrm{SS})$, the mobility of the acetabulum $(\Delta \mathrm{AI})$, and the arc of motion of the hip $(\triangle \mathrm{PFA})$. The ratio of change between sacral slope and pelvic femoral angle is that $1^{\circ}$ of SS is inversely correlated to $0.9^{\circ}$ of PFA (i.e., for every $1^{\circ}$ decrease in $\Delta \mathrm{SS}$, there is a $0.9^{\circ}$ increase in PFA motion).

The sacral slope is the most accurate measurement of dynamic change $[7,11]$. The change in sacral tilt $(\Delta \mathrm{SS})$ from standing to sitting (and vice versa) is normally $20^{\circ}$ with a normal range of $11^{\circ}-29^{\circ}$ [11]. A measurement of $<10^{\circ}$ indicates stiffness; a fused construct has a $\Delta \mathrm{SS}<$ $5^{\circ}$; and hypermobile spinopelvic construct has a $\Delta \mathrm{SS}>30^{\circ}$ [11]. Pelvic femoral angle (PFA) is the measure of femoral motion, and the mean is $180^{\circ}$ standing and $125^{\circ}$ sitting. This femoral motion is more important for impingement than the acetabulum.

Ante-inclination is a sagittal measurement of the acetabulum that can be measured in the preoperative hip as well as the cup position following THA [7]. Ante-inclination is correlated to the coronal cup inclination and anteversion. The normal range for standing ante-inclination following THA is $42^{\circ}-63^{\circ}$, which provides a sagittal safe zone for acetabular cup position which can be used to help estimate the risk of impingement [11].

Two other measurements are used. The first is the coronal combined anteversion of the stem and cup which is important because both sides of the joint must be included to fully understand hip impingement and stability [12]. The second measurement, Combined Sagittal Index, is a measure of the sagittal hip motion, and it predicts risk of and direction of dislocation. The combined sagittal index (CSI) is the sum of AI and PFA with outliers of standing values of $\geq 243^{\circ}$ and sitting values of $\leq 151^{\circ}$. This measurement can be considered the functional hip motion safe zone because it is: $\mathrm{CSI}=\mathrm{AI}+\mathrm{PFA}$.

\subsection{Abnormal Spine-Hip-Pelvis Motion}

Spinopelvic-hip imbalance is hypermobility or stiffness of the spinopelvic construct. When the pelvic motion during postural change is altered from normal, it impacts the sagittal acetabular angle (ante-inclination), and to compensate for abnormal pelvic motion, the femur as measured by the PFA must also change. Stiffness is almost always caused by degenerative disc disease or fusion surgery [11]. In a study of 160 patients, $30 \%$ of those below the age of 60 years had radiographic stiffness of the spine, while $55 \%$ of patients older than 60 were affected [11].

Stiffness of the spinopelvic construct means the sacral slope moves $\leqq 10^{\circ}$ with postural change between standing and sitting. But imbalance can also be categorized into patterns, and patterns help us understand how to compensate for spinopelvic abnormality when performing total hip replacement. Within a pattern, motion may or may not be stiff. Stefl et al. [11] define specific patterns of imbalance and the effect on acetabular position. Stefl's patterns are defined by the position in which the spinopelvic construct is fixed in both the standing and sitting postures. In the standing position, the pelvis is fixed in anterior tilt. Therefore, the subset of patients with posterior tilt $<30^{\circ}$ with sitting is classified as stuck-standing. With this pattern, the acetabulum does not completely open during sitting (and the less mobility of sacral slope between standing and sitting, the less it opens), so the hip has to flex more to allow sitting which increases the risk of anterior impingement of the greater trochanter on the pelvis [20, 22]. Conversely with stuck sitting, the pelvis is fixed in posterior tilt. In a subset of patients, it does not tilt anteriorly $>30^{\circ}$ with standing, and these patients are thus classified as stuck sitting [11]. Here, the femur must hyperextend (increased PFA) for the person to stand up straight, and the risk is posterior impingement of the greater trochanter on the pelvis, and lesser trochanter on the ischium.

There is a pattern of hypermobility $\left(\Delta \mathrm{ST}>30^{\circ}\right)$ that we consider a normal variant, and is found mostly in younger age and women 
patients. Hypermobility is considered to be imbalance when the increased mobility of the spine is a result of the spine tilting beyond flat with sitting. Kyphosis as a spinopelvic motion pattern occurs when hips are so stiff they do not bend enough to allow sitting so the pelvis must tilt excessively posteriorly. This is most common in patients with stiffening collagen vascular disease and in patients with a BMI over 40 because the trunk mass, with sitting, forces the balance center posteriorly.

Spinopelvic-hip imbalance occurs in $40 \%$ of patients who are undergoing primary THA [11]. The occurrence of each pattern of spinopelvic imbalance has been determined [11]. Stiffness alone $\left(<10^{\circ} \mathrm{ST}\right.$ mobility) without a pattern is $3 \%$, and each of the stuck standing and stuck sitting patterns are $14 \%$. Kyphotic deformity occurs in $11 \%$ of patients $[11,23,24]$.

\subsection{Clinical Significance of Spinopelvic-Hip Imbalance}

The loss of the smooth transition of the spinepelvis-hip movement can cause hip impingement, and with THA can affect the usual cup positions the surgeon selects. Patients presenting for primary THA with normal spine-pelvis-hip mobility are at low risk of prosthetic impingement with cups that are: in the Lewinnek safe zones [2], combined anteversion safe zone [12], and have restoration of the physiological biomechanical balance (center of rotation, hip length, and offset). In these patients, hip impingement is caused by component malposition (prosthetic impingement) or a short hip length and/or offset (extra-articular impingement) [13]. Sadhu et al., [14] recently confirmed that dislocations in primary THA occur more often in hips with cups outside the Lewinnek safe zones. However, correct coronal cup position does not always protect patients with sagittal hip motion outside the normal range. Our data shows $14 \%$ of hips having the cup aligned inside the Lewinnek zone are not in the normal sagittal hip motion zone. The primary predictive factor is increased hip motion, the second is a stiff Sacral Slope $\left(\Delta \mathrm{SS} \leq 10^{\circ}\right)$ and the third is low pelvic incidence. We agree with the futility of the Lewinnek safe zone and have declared it as meaningless with the functional safe zone more predictable [23, 24]. Pathologic stiffness is the biggest threat for impingement because of increased hip flexion, and the classic example is patients with a surgical spinal fusion who are known to have increased risk of dislocation [15].

\subsection{What Does Imbalance Mean for THA?}

Surgeons are used to viewing the hip replacement on coronal radiographs, and the science of sagittal cup position during postural change is new. Its contribution to the understanding of impingement with THA, which is a silent source of failure, is also new [4, 7, 11]. Surgeons cannot easily diagnose impingement because it is a clinical diagnosis, and no imaging or computer technique is available to identify it. Dislocation is the most recognized consequence, and it occurs when the collision of impingement, either component or bony, is severe enough that the mechanical constraint of the combined anteversion of stem and cup, and the biological constraint of the capsule and muscle tension, cannot prevent escape at the egress site [16], but other complications occur because of impingement. Pain is a known consequence but difficult to classify; wear debris and fluid in the joint may or may not be symptomatic or destructive (pseudotumors can be destructive) [17]; loosening of components occurs because of constant collision of impingement [16].

To reduce the risk of impingement with spinal imbalance, the THA cup must be positioned to compensate for sagittal change of acetabular mobility and maintain the hip inside the functional safe zone. It is for this reason that the suggestion has been made that personalization of the cup position for each patient with spinopelvic-hip imbalance is preferable $[8,11,18]$. The cup positions that keep the sagittal motion of the cup in the ante-inclination safe zone for each pattern of imbalance have been defined by Stefl et al. [11]. 
Cup inclination and anteversion used for normal pelvic motion is satisfactory for hips that are stuck sitting or stuck standing if the ST motion within that pattern is $>10^{\circ}$. The dangerous hips are those that are both fixed with anterior or posterior tilt and have a stiff ST. The functional hip safe zone cannot be duplicated with use of femoral head size, risk of wear with inclination $>45^{\circ}$, or the anterior hip approach. This creates the conundrum that the anatomic acetabular position cannot be used for the THA cup in patients with severe spinopelvic imbalance. With spinopelvic stiffness, the coronal inclination and anteversion of the cup need to be higher to mechanically open the cup, and with hypermobility the position needs to be more closed, so that it does not open too much.

Patients who have revision THA or late dislocations are older than those with primary THA and have a greater prevalence of spinopelvic stiffness. Pathologic stiffness creates risk of dislocation for both primary and revision THA, but in a study of patients 10 years after THA there were $60 \%$ with spinopelvic stiffness compared to $20 \%$ in those undergoing primary THA [11, 19]. In primary THA, the hips with dangerous stiffness can be controlled with a mechanically opened cup because the capsule adds biological constraint. In older patients with revision THA and those with late dislocation, dangerous stiffness is related to dislocation in $70-90 \%$ of cases because the impingement risk is compounded by loss of capsular integrity and abductor muscle strength [20].

\subsection{Technical Changes for Spinopelvic Imbalance}

Preoperative planning for patients with spinopelvic imbalance requires obtaining lateral spinopelvichip X-rays (see Case Report). But how does the surgeon define those patients? The easiest way is to X-ray all patients which we do. If selection is preferred, we recommend patients over 65 years of age; those with prior spine surgery; those with symptoms of stenosis; and particularly those with increased PFA in either direction [11, 23, 24]. From these stand and sit sagittal X-rays, the cup position can be planned according to the combined mobility and position of the spinopelvic construct as summarized in the previous section. Specific numbers are published according to this combination of the spinopelvic construct. Our data shows $14 \%$ of hips have no safe zone (even if inside the Lewinnek zone) because they are not within the functional safe zone, which is defined by the sum of the cup ante-inclination (AI) + PFA, and this is named the Combined Sagittal Index (CSI) [20, 23]. And this percentage of functional outliers was in a group of patients where $92 \%$ of hips were in the Lewinnek zones because we used computer navigation. The number of outliers might be higher if the percentage inside the Lewinnek zones were only 50\% [21]. They are identified best on sitting sagittal X-rays and have low PFA (increased femoral flexion) as the primary predictor for outlier of CSI but are even more at risk if there is a stiff sacral slope combined with increased hip motion. Low PI was the third most prevalent predictor, and cup position is not in the top three. But the cup counts because these hips need optimal coronal cup position to optimize AI (which is part of CSI equation). Hips with abnormal PFA, $\Delta \mathrm{SS}<10^{\circ}$ and low PI have no functional safe zone so should have additional mechanical support of dual mobility articulation at surgery because biological balance cannot be obtained.

Intraoperatively, we prepare the femur first because combined anteversion is more important than anteversion itself. If femoral anteversion is less than $5^{\circ}$ to retroverted, a decision to change the femur to a modular design or cement the femoral stem at $10^{\circ}$ anteversion (any more anteversion results in intoeing for patients) must be made. The cup is targeted to be within the combined anteversion safe zone of $25^{\circ}-45^{\circ}$ with stiff spinopelvic hips having a higher cup anteversion and thus a higher combined anteversion. Retroverted hips are not able to be anteverted sufficiently, so a decision must be made if the articulation is stable or a dual mobility is needed. Retroverted hips can be diagnosed preoperatively by the X-ray signs of crossover or ischial spine 
sign. Intraoperatively, the noncemented stem anteversion in the femur is $5^{\circ}$ or less (and if zero or less, it is best to cement the stem), and the cup is difficult to anteversion more than $10^{\circ}$ without prominent metal above the posterior rim of the acetabulum. Patients with pincer impingement commonly have retroversion of the acetabulum. If noncemented implants are chosen, it is better to use dual mobility articulation to increase mechanical stability. We use computer navigation to be accurate in cup positioning, but if the surgeon decides not to use a smart tool then he/ she must validate the precision of their manual technique. This can be done by measuring the postoperative sagittal X-ray to verify the hip is within the normal CSI and the femoral head is centralized in the cup on the sitting X-ray.

\subsection{Summary}

The literature on spine-pelvic-hip mobility and its effects on THA continue to grow. It is important for hip surgeons to begin considering the spinopelvic construct in preoperative planning, intraoperative technique, and postoperative risk stratification. Understanding the patterns of spine-pelvic-hip anatomy and mobility helps the hip surgeon to optimize and personalized cup position. The Lewinnek safe zone [2] has been used for decades, but it is well known that dislocations occur both inside and outside that safe zone [14]. Its importance has been as a guide to improve our precision of cup placement. Likewise, the use of spinopelvic imbalance will guide hip surgeons toward proper patient-specific component positioning by focusing on the functional hip motion safe zone. The authors recommend performing lateral spinopelvic X-rays on all preoperative THA patients because recent data shows $40 \%$ of primary patients have spinal imbalance. Lateral spinopelvic X-rays from L3 to $\mathrm{S} 1$ are sufficient, and full-length films like EOS are not necessary because it is the lower lumbar segment (L3-S1) that is connected to the hip.

Spinopelvic mobility affects the dynamic cup position and should be considered during posi- tioning of the acetabular cup. Consideration of the specific spinopelvic motion patterns described in this chapter can minimize the risk of impingement. In patients with spinopelvic hypermobility, the coronal cup position must be more closed so that the acetabulum does not open excessively with sitting. Conversely, if there is stiffness, the cup should be opened to prevent impingement with sitting. In the positional patterns of stuck standing or sitting without mobility stiffness, inclination of $40^{\circ}$ and anteversion of $20^{\circ}$ will almost always keep the sagittal cup position in the AI safe zone if the pattern is not stiff too. In the patient undergoing primary THA with pathologic spinal imbalance (fused spine or kyphosis with fixed posterior pelvic tilt), surgeons should consider adding constraint to the THA with a dual mobility articulation. It is also important to remove any bony impingement of the greater and lesser trochanter in patients with stiff constructs because mechanical constraint alone does not always provide sufficient protection from dislocation. This is necessary when stuck standing or stuck sitting pattern with stiffness is present (see Case Report). There is sufficient bone both anteriorly and posteriorly on the greater trochanter to do this with a high-speed drill. If this treatment will injure the gluteus medius, then the trochanter should be transferred.

\section{Case Report}

An 80-year-old female presented to our clinic after having two anterior dislocations out of her right total hip arthroplasty. She underwent an uncomplicated right total hip arthroplasty approximately 19 years ago and a left total hip arthroplasty 4 years ago, both through a posterior approach. Her left total hip arthroplasty has been asymptomatic since the time of surgery. However, her right total hip arthroplasty has dislocated twice- the first dislocation occurred approximately 15 years after her index procedure and was treated successfully with a closed reduction and; the second dislocation occurred approximately 19 years after surgery, and she underwent 
a closed reduction at an outside hospital. Both occurred while walking without any precipitating traumatic events. The patient reported apprehension while walking and intense fear of repeat dislocation that hindered her from performing daily activities.

Standard anteroposterior pelvic radiographs revealed excessive polyethylene wear and a wellfixed cementless acetabular cup and femoral stem on the right side (Fig. 12.3). Standing and sitting lateral spine-pelvis-hip radiographs revealed stiff spinopelvic motion as indicated by a delta sacral slope of $10^{\circ}$ (Fig. 12.4). Given these radiographic findings, as well as her two previous dislocations, we recommended revision surgery to restore stability. At the time of revision surgery, the patient was found to have wear debris in her capsule that was debrided. The cup was measured using computer navigation and found to have $55^{\circ}$ of inclination and $14^{\circ}$ of anteversion. Her stem and cup were found to be well fixed and left in place; she was revised with a constrained liner and had excellent stability throughout a functional arc of motion (Fig. 12.5). However,
6 weeks following her revision surgery, she dislocated anteriorly while walking.

The patient underwent a second revision surgery. At the time of surgery, the patient's constrained ring was found to be dissociated from her liner. The patient's acetabular cup was removed, and a new acetabular cup was placed with a new

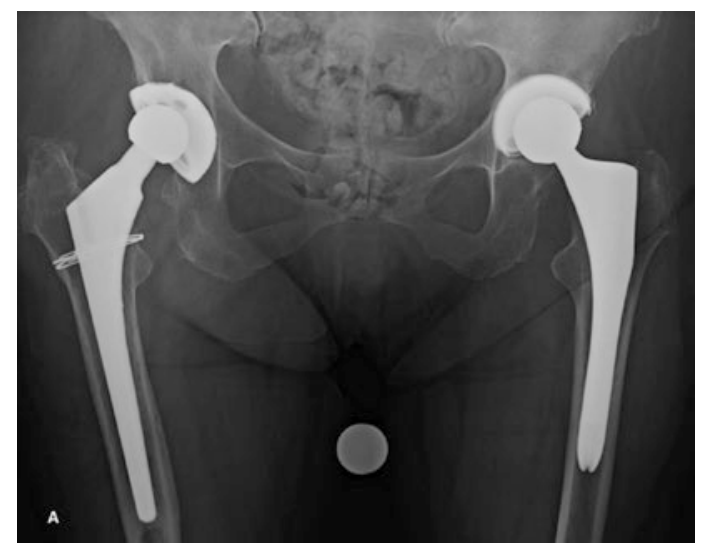

Fig. 12.3 Standard low AP pelvis of patient upon presentation. Note the excessive polyethylene wear and vertically oriented cup on the right side

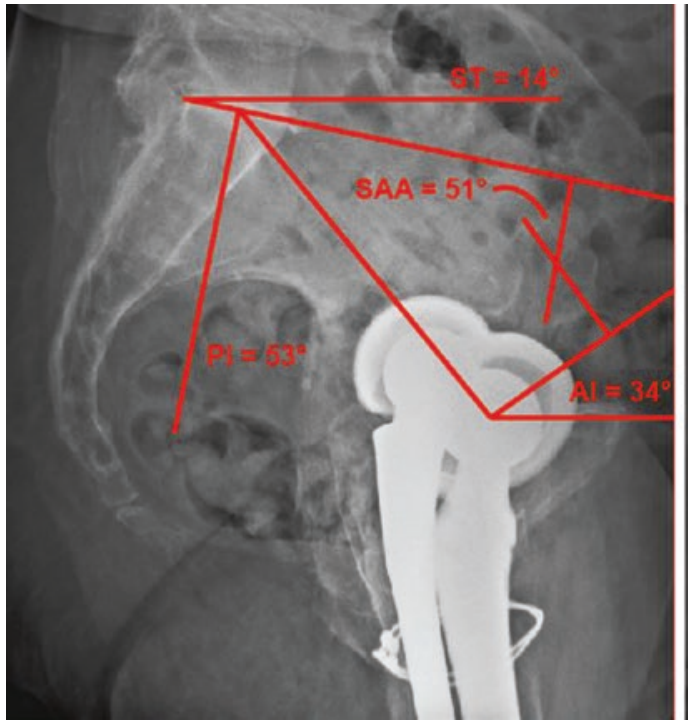

Fig. 12.4 Lateral standing and sitting spine-pelvis-hip radiographs, demonstrating a posteriorly tilted pelvis as indicated by a standing sacral tilt of $14^{\circ}$. Also, the change

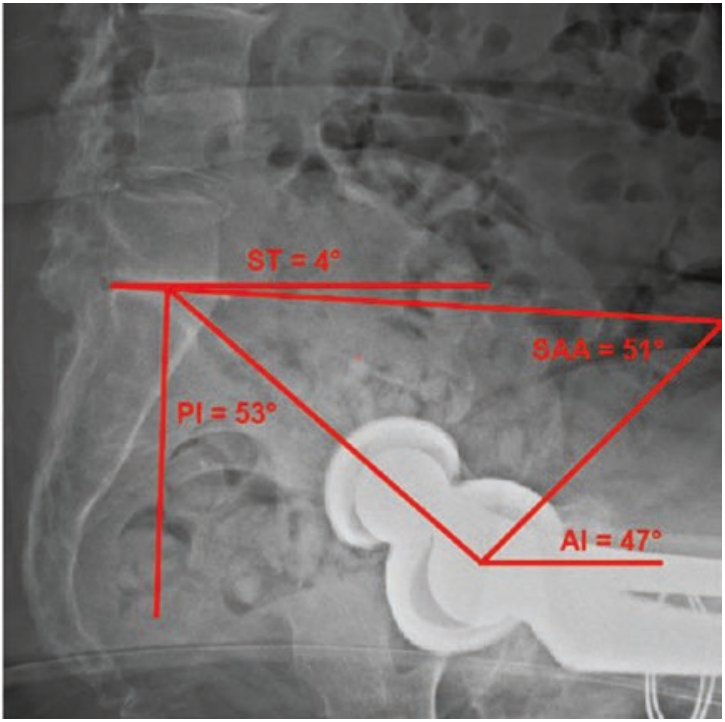

in sacral tilt $(\Delta \mathrm{ST})$ is only $10^{\circ}$, indicating decreased spinopelvic motion 


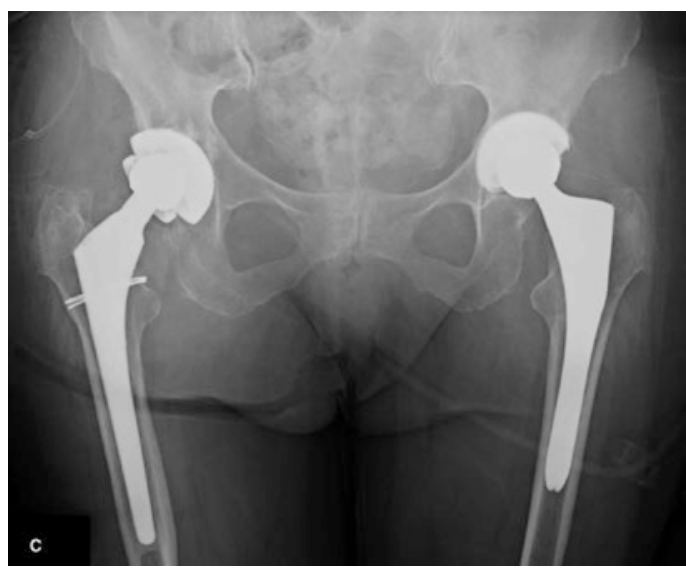

Fig. 12.5 Standard low AP pelvis, following first revision surgery to a constrained liner

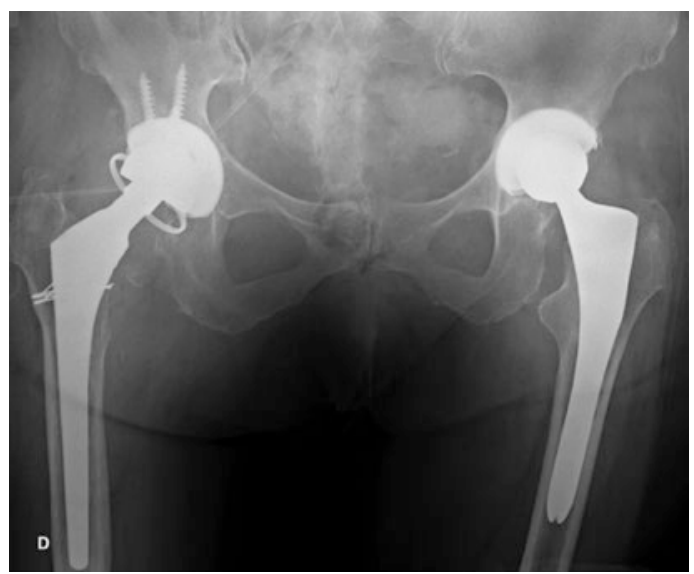

Fig. 12.6 Standard low AP pelvis, following second revision surgery with replacement of the acetabular cup and implantation of a new constrained liner

constrained liner and $+10-\mathrm{mm}$ increased head length (Fig. 12.6). The patient's hip was stable throughout a functional arc of motion; however, with terminal extension and external rotation of her femur, her greater trochanter impinged on her ilium, and her lesser trochanter impinged on her ischium. To avoid future impingement, her lesser trochanter and the posterior portion of her greater trochanter were removed with care taken to not violate the gluteus medius tendon (Fig. 12.7). The patient had an uneventful recovery and had no further episodes of instability at her 2-year follow-up.

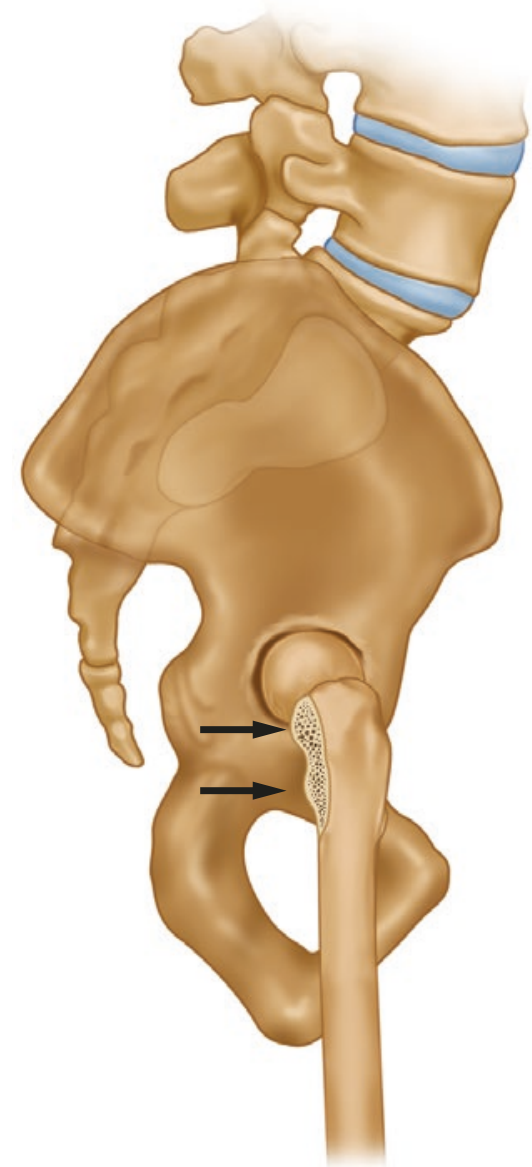

Fig. 12.7 An illustration depicting the portion of the greater trochanter, typically removed to avoid impingement. If the amount of bony excision required to avoid impingement is thought to disrupt the gluteus medius tendon, a trochanteric transfer can be performed

\section{References}

1. Lewinnek GE, Lewis JL, Tarr R, Compere CL, Zimmerman JR. Dislocations after total hipreplacement arthroplasties. J Bone Joint Surg Am. 1978;60(2):217-20.

2. Murray DW. The definition and measurement of acetabular orientation. J Bone Joint Surg Br. 1993;75:228-32.

3. DiGioia AM, Jaramaz B, Blackwell M, Simon DA, Morgan F, Moody JE, Nikou C, Colgan BD, Aston CA, Labarca RS, Kischell E, Kanade T. The Otto Aufranc Award. Image guided navigation system to measure intraoperatively acetabular implant alignment. Clin Orthop Relat Res. 1998;355:8-22. 
4. Lazennec JY, Charlot N, Gorin M, Roger B, Arafati N, Bissery A, Saillant G. Hip-spine relationship: a radioanatomical study for optimization in acetabular cup positioning. Surg Radiol Anat. 2004;26(2):136-44.

5. Philippot R, Wegrzyn J, Farizon F, Fessy MH. Pelvic balance in sagittal and Lewinnek reference planes in the standing, supine and sitting positions. Orthop Traumatol Surg Res. 2009;95(1):70-6.

6. Legaye J, Duval-Beaupère G, Hecquet J, Marty C. Pelvic incidence: a fundamental pelvic parameter for three-dimensional regulation of spinal sagittal curves. Eur Spine J. 1998;7(2):99-103.

7. Kanawade V, Dorr LD, Wan Z. Predictability of acetabular component angular change with postural shift from standing to sitting position. J Bone Joint Surg Am. 2014;6(12):978-86.

8. Phan D, Bederman SS, Schwarzkopf R. The influence of sagittal spinal deformity on anteversion of the acetabular component in total hip arthroplasty. Bone Joint J. 2015;97-B(8):1017-23.

9. Larkin B, van Holsbeeck M, Koueiter D, Zaltz I. What is the impingement-free range of motion of the asymptomatic hip in young adult males? Clin Orthop Relat Res. 2015;473(4):1284-8.

10. Sugano N, Tsuda K, Miki H, Tako M, Suzuki N, Nakamura N. Dynamic measurements of hip movement in deep bending activities after total hip arthroplasty using a 4-dimensional motion analysis system. J Arthrop. 2012;27:1562-8.

11. Stefl M, Lundergan W, Heckmann N, McKnight B, Ike H, Murgai R, Dorr LD. Spinopelvic mobility and acetabular component position for total hip arthroplasty. Bone Joint J. 2017;99-B(1 Supple A):37-45.

12. Dorr LD, Malik A, Dastane M, Wan Z. Combined anteversion technique for total hip arthroplasty. Clin Orthop Relat Res. 2009;467(1):119-27.

13. Dorr LD, Wan Z. Causes of and treatment protocol for instability of total hip replacement. Clin Orthop Relat Res. 1998;355:144-51.

14. Sadhu A, Nam D, Coobs B, Barrack TN, Nunley RM, Barrack RL. Acetabular component position and the risk of dislocation following primary and revision Total hip arthroplasty: a matched cohort analysis. J Arthroplast. 2017;32:987-91.
15. Buckland AJ, Hart RA, Mundis GM Jr, et al. Risk of total hip arthroplasty dislocation after adult spinal deformity correction. Spine J. 2016;16(10):S180. https://doi.org/10.1016/j. spinee.2016.07.086.

16. Brown TD, Elkins JM, Pedersen DR, Callaghan JJ. Impingement and dislocation in total hip arthroplasty: mechanisms and consequences. Iowa Orthop J. 2014;34:1-15.

17. Grammatopoulos G, Pandit H, Kwon YM, Gundle R, McLardy-Smith P, Beard DJ, Murray DW, Gill HS. Hip resurfacings revised for inflammatory pseudotumour have a poor outcome. J Bone Joint Surg Br. 2009;91(8):1019-24.

18. Pierrepont J, Hawdon G, Miles BP, Connor BO, Baré J, Walter LR, Marel E, Solomon M, McMahon $\mathrm{S}$, Shimmin AJ. Variation in functional pelvic tilt in patients undergoing total hip arthroplasty. Bone Joint J. 2017;99-B(2):184-91.

19. Yukizawa Y, Dorr LD, Ward JA, Wan Z. Posterior mini-incision with primary total hip arthroplasty: a nine to ten year follow up study. J Arthroplast. 2016;31(1):168-7.

20. Heckmann N, Stefl M, Trasolini N, McKnight B, Ike H, Dorr LD. Late dislocation following total hip arthroplasty. JBJS. 2018;100:1845-53.

21. Callanan MC, Jarrett B, Bragdon CR, Zurakowski D, Rubash HE, Freiberg AA, Malchau H. The John Charnley Award: risk factors for cup malpositioning: quality improvement through a joint registry at a tertiary hospital. Clin Orthop Relat Res. 2011;469(2):319-29.

22. Ike H, Dorr LD, Trasolini N, Stefl M, McKnight B, Heckmann N. Spine-pelvis-hip relationship in THR functioning of a total hip replacement. J Bone Joint Surg Am. 2018;100:1606-15.

23. Tezuka T, Heckmann N, Bodner R, Dorr LD. Functional safe zone is superior to the Lewinnek safe zone for total hip arthroplasty: why the Lewinnek safe zone is not always predictive of stability. J Arthroplasty. 2019;34:3-8.

24. Dorr LD, Callaghan JJ. Death of the Lewinnek "safe zone". J Arthroplasty. 2019;34:1-2.

Open Access This chapter is licensed under the terms of the Creative Commons Attribution 4.0 International License (http://creativecommons.org/licenses/by/4.0/), which permits use, sharing, adaptation, distribution and reproduction in any medium or format, as long as you give appropriate credit to the original author(s) and the source, provide a link to the Creative Commons license and indicate if changes were made.

The images or other third party material in this chapter are included in the chapter's Creative Commons license, unless indicated otherwise in a credit line to the material. If material is not included in the chapter's Creative Commons license and your intended use is not permitted by statutory regulation or exceeds the permitted use, you will need to obtain permission directly from the copyright holder. 